\title{
Lack of Respiratory Coupling with Neocortical and Hippocampal Slow Oscillations
}

\author{
Jeremy Viczko, ${ }^{1}$ Arjun V. Sharma, ${ }^{1}$ Silvia Pagliardini, ${ }^{2,3}$ Trish Wolansky, ${ }^{3}$ and Clayton T. Dickson ${ }^{1,2,3}$ \\ ${ }^{1}$ Department of Psychology, ${ }^{2}$ Department of Physiology, and ${ }^{3}$ Centre for Neuroscience, University of Alberta, Edmonton, Alberta, Canada T6G 2E9
}

\begin{abstract}
Previous work has demonstrated an influence of the respiratory cycle and, more specifically, rhythmic nasal inspiration for the entrainment of slow oscillations in olfactory cortex during ketamine-xylazine anesthesia. This respiratory entrainment has been suggested to occur more broadly during slow-wave states (including sleep) throughout the forebrain, in particular in the frontal and parahippocampal and hippocampal cortices. Using multisite local field potential recording methods and spectral coherence analysis in the rat, we show here that no such broad forebrain coupling takes place during slow-wave activity patterns under either ketamine-xylazine or urethane anesthesia and, furthermore, that it also does not arise during natural slow-wave sleep. Therefore, respiratory-related oscillatory neural activities are likely limited to primary olfactory structures during slow-wave forebrain states.
\end{abstract}

Key words: breathing; local field potential; olfactory rhythm; sleep; slow-wave sleep; spontaneous brain activity

\section{Introduction}

Neuronal population dynamics, the coordinated spatiotemporal activities of cell assemblies in the brain, are an important feature of the online function of the nervous system in the ongoing regulation of behavior (Buzsáki and Draguhn, 2004; Buzsáki, 2006). Conversely, the spontaneous coordination of neural activity occurring in offline states such as sleep has also been suggested to be important for the modulation and consolidation of neuroplastic changes occurring during prior wakefulness, which would have an impact on future online brain operations and behavior (Buzsáki, 1989, 1998; Born et al., 2006). Patterns occurring during non-rapid-eye movement (nREM) stages (especially slow-wave-sleep [SWS]) are thought to be especially important in this regard (Born et al., 2006; Tononi and Cirelli, 2006; Dickson, 2010; Girardeau and Zugaro, 2011; Mölle and Born, 2011). Therefore, the mechanisms by which spontaneous globally occurring slow-wave patterns are generated and entrained are of fundamental importance.

The slow oscillation (SO) is an archetypal sleep EEG pattern expressed during nREM and especially SWS. It is a slowly occurring $(\leq 1 \mathrm{~Hz})$, global forebrain EEG pattern that is also present during ketamine or urethane anesthesia (Steriade et al., 1993a). At the cellular level, it corresponds to the synchronous fluctuation of membrane potential from relatively hyperpolarized levels

Received Aug. 19, 2013; revised Jan. 17, 2014; accepted Jan. 24, 2014.

Author contributions: C.T.D. designed research; J.M.V., A.V.S., S.P., and T.W. performed research; J.M.V. contributed unpublished reagents/analytic tools; J.M.V., A.V.S., and T.W. analyzed data; J.M.V. and C.T.D. wrote the paper.

This work was supported by the Natural Sciences and Engineering Research Council of Canada (Grant RGPIN 249861 to C.T.D.) and the Branch Out Neurological Foundation (summer studentship funding to J.M.V.). C.T.D. is an Alberta Ingenuity Health Solutions Senior Scholar. We thank Alyssa Hindle and Anastasia Greenberg for assistance with experiments and analyses, respectively.

The authors declare no competing financial interests.

Correspondence should be addressed to Clayton T. Dickson, Department of Psychology, University of Alberta, Biological Sciences Building, P439, Edmonton, Alberta, Canada T6G 2E9. E-mail: clayton.dickson@ualberta.ca.

DOI:10.1523/JNEUROSCI.3581-13.2014

Copyright $\odot 2014$ the authors $\quad 0270-6474 / 14 / 343937-10 \$ 15.00 / 0$
(DOWN/OFF epochs) to suprathreshold levels (UP/ON epochs) (Steriade et al., 1993b). The SO, in particular, has been associated with future memory function (Marshall and Born, 2007; Dickson, 2010; Mölle and Born, 2011). Although it is typically thought of as an intrinsic spontaneous rhythm of cortical networks because it is generated in the isolated forebrain, in isolated cortical slabs, and may also be a component of activity present in in vitro preparations (Sanchez-Vives and McCormick, 2000; Timofeev et al., 2000; Dickson et al., 2003), it is also elicited or influenced by sensory stimulation, electric/magnetic fields, corticocortical interconnections, and direct neural stimulation, suggesting that its global coordination is entrained by activity-dependent processes (Amzica and Steriade, 1995; Petersen et al., 2003; MacLean et al., 2005; Massimini et al., 2007; Gao et al., 2009; Vyazovskiy et al., 2009; Ozen et al., 2010; Zhang et al., 2011; Greenberg and Dickson, 2013).

On the basis of recordings made from the piriform cortex (PIRI) in ketamine-xylazine anesthetized rats, a previous and seemingly untested idea has been that the $\mathrm{SO}$ occurring throughout the rest of the cortex (including paleocortices, archicortices, and neocortices) may be influenced and entrained by rhythmic respiratory related activity deriving from olfactory circuits (Fontanini et al., 2003; Fontanini and Bower, 2006). In supplementary neocortical and hippocampal recordings (Wolansky et al., 2006), we found no respiratory cycle coupling to the SO expressed during nREM-like states. However, to date, there have been no systematic and explicit evaluations of specific respiratory coupling to the $\mathrm{SO}$ at other forebrain sites beyond olfactory cortex, nor has this been assessed across a wider array of conditions, including ketamine/urethane anesthesia or natural sleep. In this study, we demonstrate a complete lack of respiratory-EEG coupling during SO activity across all slow-wave conditions tested except at the level of piriform (olfactory) cortex. Interestingly, and in contrast to previous work (Fontanini and Bower, 2005), we found here that forebrain coupling to respiratory frequencies was enhanced 
during activated states (REM and REM-like) states during natural sleep and urethane anesthesia. Regardless, sleep-related forebrain $\mathrm{SO}$ in cortical structures outside of primary olfactory areas is entirely independent of rhythmic respiratory activity.

\section{Materials and Methods}

Animals. Data were collected from 24 male Sprague Dawley rats (weight: $302 \pm 2.9 \mathrm{~g}$ ). All procedures followed the guidelines established by the Canadian Council on Animal Care as well as the Society for Neuroscience. This protocol was also approved by the Biological Sciences Animal Policy and Welfare Committee of the University of Alberta.

Anesthetized (ketamine/xylazine and urethane) preparations. Data were collected from 12 ketamine/xylazine-anesthetized (KET) and eight urethane-anesthetized (URE) rats. Animals were induced with gaseous isoflurane mixed with medical oxygen at a minimum alveolar concentration (MAC) of 4.0 in a Plexiglas anesthetic chamber. After the loss of righting reflexes, rats were transferred to a nose cone delivering isoflurane at a MAC of 1.5-2.5. A catheter was inserted into the jugular vein and rats were switched from isoflurane anesthesia to intravenous ketamine/xylazine $(60 / 6 \mathrm{mg} / \mathrm{kg}$, concentration $100 / 10 \mathrm{mg} / \mathrm{ml})$ or urethane $(1.7 \mathrm{~g} / \mathrm{kg}$; concentration $0.7-0.8 \mathrm{~g} / \mathrm{ml})$ via the jugular catheter. In some cases, the same ketamine-xylazine mixture was administered intraperitoneally $(100 / 10 \mathrm{mg} / \mathrm{kg})$. In all cases, the anesthetic level was assessed using hindpaw pressure. Additional doses of the particular anesthetic specific to each condition were delivered as appropriate to maintain a surgical plane of anesthesia. Body temperature was maintained constant at $37^{\circ} \mathrm{C}$ using a servo-driven system connected to a heating pad and rectal probe (TR-100; Fine Science Tools).

Rats were positioned in a stereotaxic frame and prepared for intracranial recordings. The coordinates for implantation sites across all subjects were measured relative to bregma and are reported in millimeters. Monopolar electrodes, constructed from Teflon-coated stainless steel wire with $125 \mu \mathrm{m}$ diameter (A-M Systems), were targeted at frontal cortical regions (nCTX: AP, +1.5 to 2.5 ; ML 2.2 to 2.6 ; DV -1.25 to -1.7 ) and hippocampus (HPC: AP -3.3; ML 2.4; DV -2.5 to -3.0 ). In some cases (KET, $n=3$; URE, $n=4)$, bipolar electrodes $(0.7-1.5 \mathrm{~mm}$ tip separation) were also positioned in the pirifrom cortex (PIRI: AP + 1.0; \pm ML 4.5; DV -8.3 to -8.9 ). In a further subset of animals under URE, we implanted similar bipolar electrodes in the hippocampus. $(n=4)$.

The respiratory cycle (RESP) was measured in a variety of ways. In most cases $(n=23)$, air movements in the nasal passageway were directly monitored with a thermistor (Thermocouple Wire 30 Gauge Type K; Thermo Electric; Chaput and Holley, 1980) or by making direct measurements of airflow using an air pressure transducer (DP103-10N1S4D; Volidyne Engineering; $n=4$; Pagliardini et al., 2012). Care was taken to ensure that referencing artifacts caused by respiratory movements or grounding issues did not interfere with and were not reflected in active signals, especially for thermistor recordings. We verified (see Results section) that all recordings and analyses using different respiratory recording techniques demonstrated similar properties.

Local field potentials (LFPs) and thermistor potentials were amplified at a gain of 1000 and filtered between 0.1 and $500 \mathrm{~Hz}$ using a 4-channel AC amplifier (model 1700; A-M Systems). All monopolar recordings were referenced to the stereotaxic apparatus and bipolar recordings were referenced across electrode contacts. Airflow was measured with a pneumotach (GM Instruments) connected to a low-pressure transducer (model DP103-10; Valydine). Signals were digitized using a Digidata 1322A A-D board connected to a PC computer running Axoscope acquisition software (Molecular Devices). All signals were sampled at a rate of $1000 \mathrm{~Hz}$.

Recordings were made for a minimum of $2 \mathrm{~h}$ in all experiments. We extracted 1 min periods of profound and obvious nREM-like (SO) states during anesthesia for the main analyses and later extracted REM-like state epochs also $1 \mathrm{~min}$ in length to assess the influence of brain state changes (Wolansky et al., 2006; Clement et al., 2008; Sharma et al., 2010; Pagliardini et al., 2012). Signals from these epochs were used to analyze spectral characteristics of spontaneous activity and respiration, as well as the relationship between the two (details in Data analysis, below). After

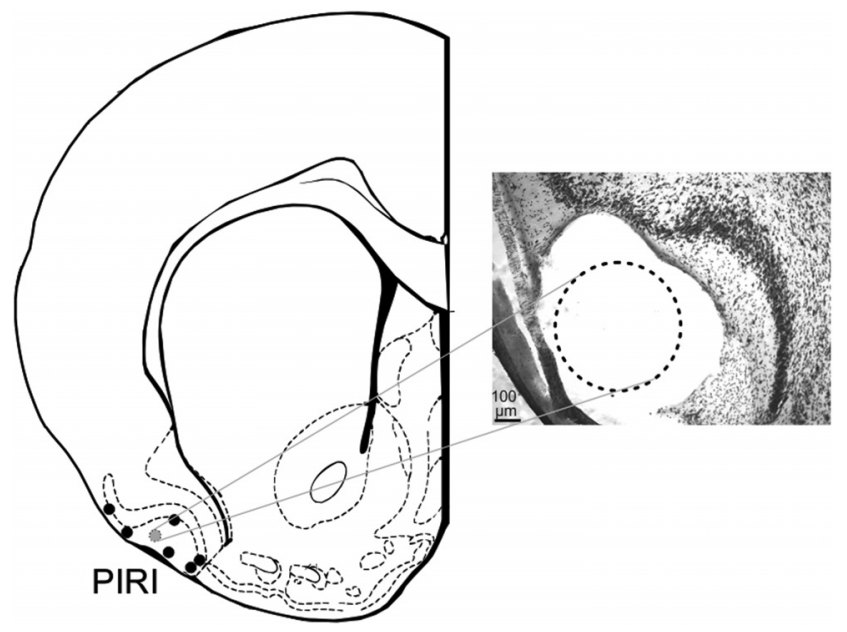

Figure 1. Histological positions of piriform recording sites. Left, Center points of the leading (deepest) electrode from each bipolar pair on a schematic diagram of a rat brain coronal section 1.00 $\mathrm{mm}$ anterior to bregma (Paxinos and Watson, 1998). Right, Photomicrographic image of the actual histology in one case (indicated by the gray circle on the left). The lesion from the longest electrode of the pair is shown with the dotted circle at the level of layer I of the piriform cortex. In all experiments, the tips of both pair of electrodes were straddling the cell layer II/III in piriform.

the recording sessions, small lesions were made by passing $100 \mu \mathrm{A} \mathrm{DC}$ current for $5 \mathrm{~s}$ (model 2100 constant current stimulator; A-M Systems) across the tips of the electrodes to later view recording sites (Fig. 1) in brain slices under a microscope.

Chronic, naturally sleeping preparation. To determine whether any coupling between respiration and cerebral activity occurred during naturally occurring SO, we obtained recordings made during natural sleep in four additional male Sprague Dawley rats that had been used in a prior study (Pagliardini et al., 2012). In brief, rats were anesthetized with an intraperitoneal injection of a ketamine-xylazine mixture $(90 \mathrm{mg} / \mathrm{kg}, 10 \mathrm{mg} / \mathrm{kg}$; concentration $100 \mathrm{mg} / \mathrm{ml}, 10 \mathrm{mg} / \mathrm{ml}$ ). Using similar stereotaxic techniques and coordinates as described for anesthetized preparations, rats were implanted with bipolar LFP electrodes at the level of frontal and hippocampal cortices and electrodes were fixed to the skull using dental acrylic and jeweler's screws fastened to the skull. Bipolar EMG electrodes were implanted in the neck (for sleep state detection) and diaphragm (DIA: to monitor inspiratory respiration) (Pagliardini et al., 2012). Wires were tunneled under the skin and attached to an electrical socket (Ginder Scientific). The socket was then fastened surgically between the shoulder blades. Rats were given an analgesic for $2 \mathrm{~d}$ after surgery (Metacam, $2 \mathrm{mg} / \mathrm{kg}$; Boehringer Ingelheim) with food and water available ad libitum and maintained on a $12 \mathrm{~h}$ dark/light cycle. During the first week after surgery, rats were habituated to the recording chamber for at least 3 sessions of $8 \mathrm{~h}$ each.

Recording sessions started $7 \mathrm{~d}$ after surgery. Rats were connected to tethering cables in the morning (10:00 A.M.) and data were collected between 12:00 P.M. and 5:00 P.M. Field potential activity was amplified at a gain of 1000 and filtered between 0.1 and $500 \mathrm{~Hz}$ using a differential AC amplifier (model 1700; A-M Systems). All signals were sampled at 1 $\mathrm{kHz}$ using a PowerLab 16/30 data acquisition system (AD Instruments; Pagliardini et al., 2012). Sleep analysis was performed on $2-3 \mathrm{~h}$ periods between 2:00 P.M. and 5:00 P.M., when rats showed consistent and robust sleep behavior. Sleep behavior was initially categorized by periods of consistently low nuchal EMG. SWS was isolated from these periods by visual inspection of records having high voltage signal fluctuations occurring between $\sim 1$ and $2.5 \mathrm{~Hz}$ with a duration of at least a full minute in both cortical and hippocampal traces. REM states were isolated by monitoring nuchal EMG for further decreases and by selecting traces showing theta activity in hippocampus (Clement et al., 2008). Activated states of wakefulness were distinguished from activated REM state and SWS by low voltage fast EEG activity concomitant with large amplitude nuchal EMG fluctuations and were not analyzed. In addition, data showing spindle, K-complex, or transition-like activity, as well as epochs with sighs in the respiratory signal were also excluded from analyses. After suffi- 


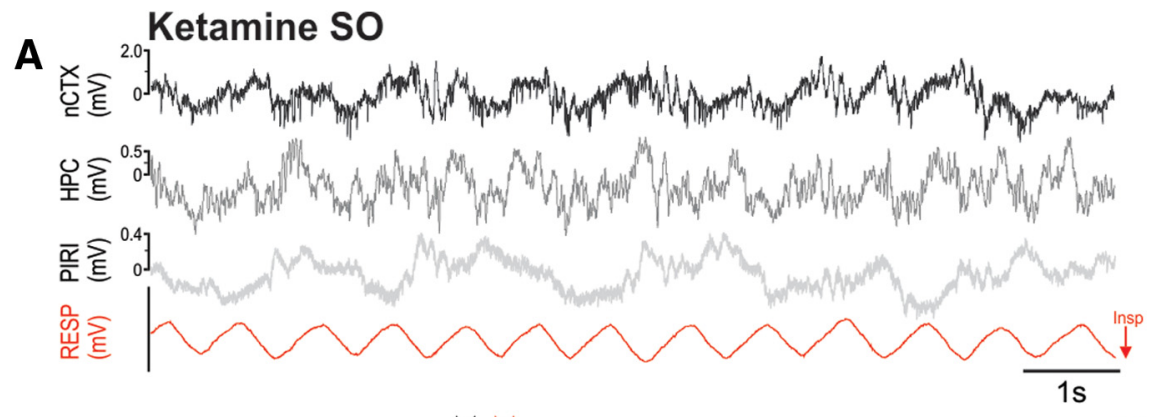

B

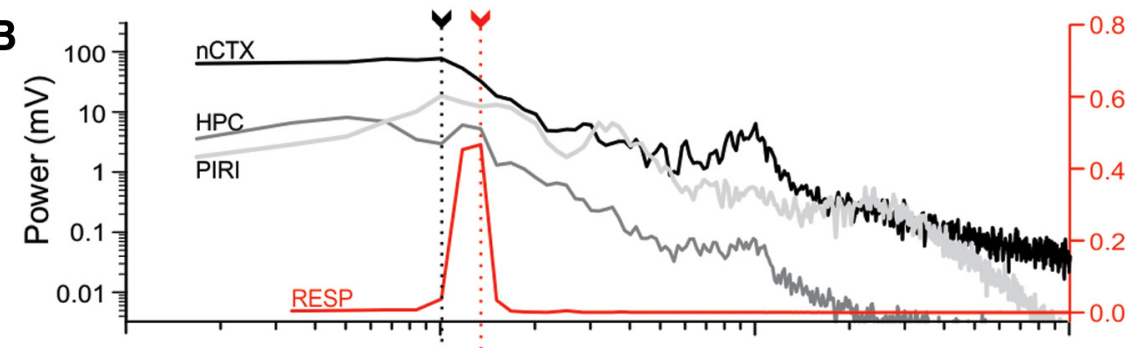

C

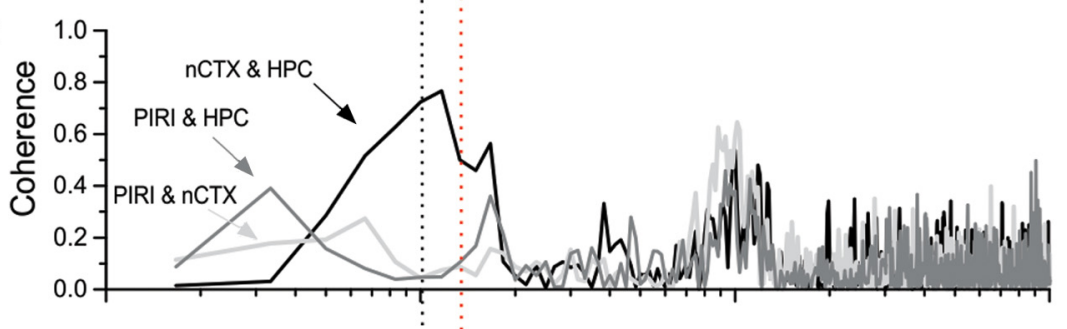

D
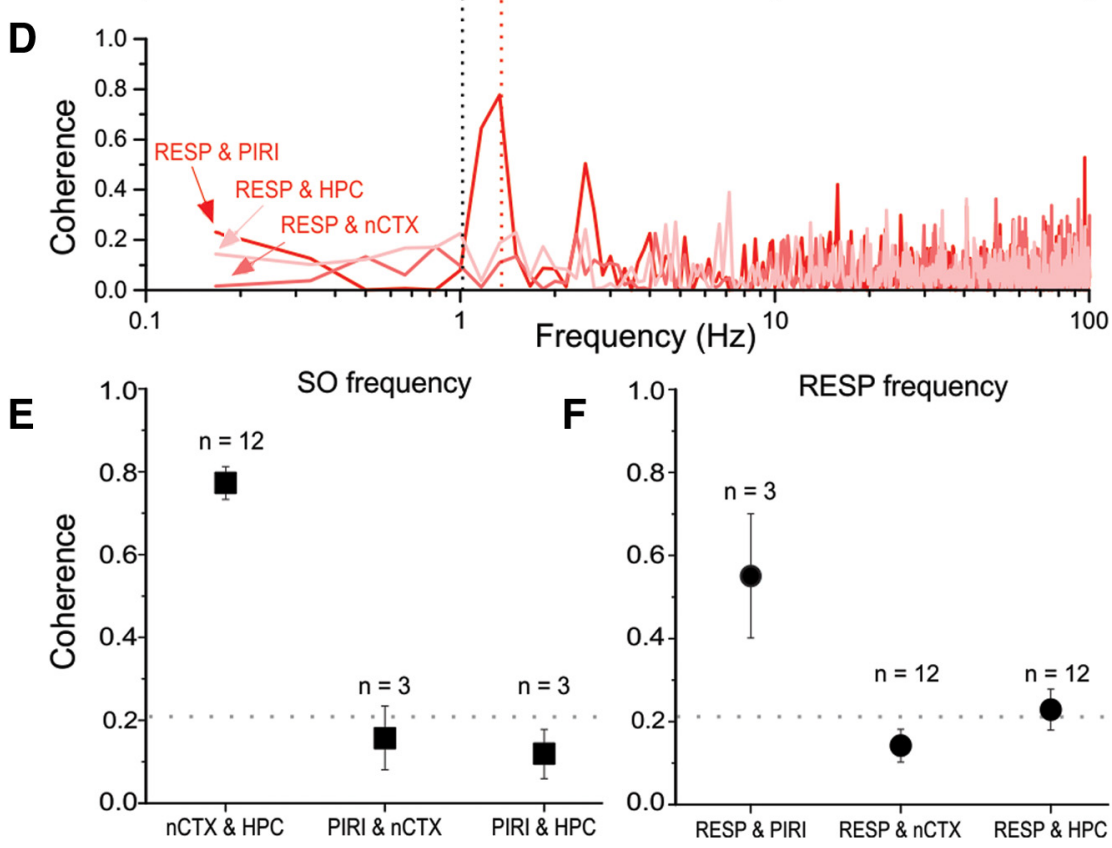

Figure 2. Lack of frequency matching of neocortical/hippocampal and respiratory signals and the lack of neocortical/hippocampal coupling to the respiratory cycle under ketamine-xylazine anesthesia. $\boldsymbol{A}$, Representativeexample of simultaneously recorded raw LFP tracesfromnCTX, HPC, and PIRIsites, togetherwith thermistorreadings from the nostril(RESP, with inspirationshownas downward deflections). $\boldsymbol{B}$,0verlaid powerspectral plots from periods (including those shown in $\boldsymbol{A}$ ) demonstrate different peak frequencies (fundamentals) when comparing brain-derived LFPs and the respiratory signal. Arrows with dotted lines indicate peak frequencies corresponding to cortical SO (black) and respiration (red) frequencies. The logarithmic scale for brain LFP power is shown on the left and the power of the respiratory signal is displayed on the linear scale to the right. Low-frequency, $<0.33 \mathrm{~Hz}$, near-DC frequencies were truncated from the respiratory plot for ease of visualization. C, Coherence spectra for brainderived LFP signal pairs, illustrating high coupling around the $S 0$ peak across neocortical and hippocampal sites. $D$, Coherence spectra for the respiratory signal to each brain LFP recording demonstrating low coupling at the respiratory fundamental except at the piriform site. All spectra shown $(\boldsymbol{B}-\boldsymbol{D})$ were computed from the same 1 min sample that included the raw sample shown in $\boldsymbol{A}$. $\boldsymbol{E}$, Average coherence $( \pm \mathrm{SEM})$ for brain-derived LFP signal pairsas noted across experiments attheSO peak. Only theHPCandnCTXtended to be coupledatSOfrequencies.F,Average coherence $( \pm$ SEM) across experiments at the respiration peakfrequency for each brain site paired to the respiratory signal. Only the piriform sitewassignificantly coupled to respiration. Forboth $\boldsymbol{E}$ and $\boldsymbol{F}$, thenumbers of subjects used in each average are displayed abovethe symbols. The horizontal dotted lineindicates the threshold for significant coherence, $>0.21$. cient recording periods, animals were deeply anesthetized before performing electrode tip lesions before histological procedures.

Histology. While maintained under deep anesthesia, rats were transcardially perfused, first using physiological saline and then with $4 \%$ paraformaldehyde in saline. Brains were extracted and placed in a solution of $30 \%$ sucrose and $4 \%$ paraformaldehyde. After a period of at least $24 \mathrm{~h}$, brain tissue was frozen with compressed carbon dioxide and then sliced using a rotary microtome (model 1320; Leica) at a thickness of $48 \mu \mathrm{m}$. The slices were then mounted on gel-coated slides and allowed to dry for at least $24 \mathrm{~h}$ before being stained with thionin and coverslipped. These stained slices were then visualized under a Leica DM LB2 microscope to verify recording sites. Digital photographs of the brain slices were taken using a Canon Powershot S45 camera before being imported into Corel PhotoPaint.

Data analysis. Raw signals were analyzed visually using AxoScope (Molecular Devices). Three $1 \mathrm{~min}$ segments of slow-wave states were extracted from the total recording for each subject under anesthesia and 5 for subjects recorded during natural sleep. In a similar fashion, REM and REM-like segments appearing in sleep and under urethane were extracted separately. Because we did not observe state changes under adequate ketamine/xylazineanesthesia, we could not perform a similar analysis in this condition. Analyses on time series signals were conducted offline using code written in MATLAB 5.3.1 (MathWorks; Wolansky et al., 2006). EMG signals were converted into a continuous respiratory cycle by performing a windowed $(250 \mathrm{~ms})$ and detrended root-mean-squared (RMS) calculation. Spectral analyses were performed using Welch's averaged modified periodogram method for power spectral density estimation and coherence ( $6 \mathrm{~s}$ window; $2 \mathrm{~s}$ overlap). These were then plotted for field signals and respiratory signals and for paired cross-channel comparisons using Origin software (Microcal Software). The significance of coherence values was assessed by comparison with a randomized coherence distribution that was computed based on a series of sequential time-shifted (and also time-reversed) coherence computations using the actual time series analyzed. The maximum $99 \%$ confidence limit for this randomized distribution (including all frequencies throughout the spectrum from 0 to 500 $\mathrm{Hz}$ ) across multiple datasets having durations of $60 \mathrm{~s}$ was found to be 0.21 . Therefore, the threshold for significant coherence was set at 0.21 for all coherence estimates.

\section{Results}

In preliminary cases, across anesthetic and natural sleep conditions, we limited our initial analysis to data acquired during periods corresponding to deactivated (slow-wave) forebrain states. To compare results across states, we also analyzed data epochs under urethane or natural sleeping 
conditions that showed REM or REM-like activity (Wolansky et al., 2006; Clement et al., 2008; Sharma et al., 2010). We verified electrode locations via histology for all PIRI recordings (Fig. 1). Bipolar PIRI recordings were classified as onsite when the leading electrode was positioned in superficial layer I with the shorter of the pair deep to layer II/III. In addition, PIRI placement was verified by time-locked LFP activity concomitant with respiratory sighs (augmented breaths; Pagliardini et al., 2012). Hippocampal placement was confirmed by auditory verification of multiunit activity in CA1 when lowering electrodes and the presence of clear theta and/or SO activity (Wolansky et al., 2006; Sharma et al., 2010). Our physiological criterion for placements was that $\mathrm{SO}$ amplitude in nCTX had to be at least $0.8 \mathrm{mV}$ peak to peak and the theta signal from HPC needed to be bigger than $0.5 \mathrm{mV}$ peak to peak. A total of three experiments were eliminated from the subsequent analyses due to electrode misplacements.

\section{Characteristics of respiratory and brain (LFP) signals}

Across all conditions, the respiratory signal (whether it was described by variations in thermistor readings, airflow output, or by the detrended RMS of diaphragm EMG activity) was characterized by a near sinuisoidal waveform (Figs. 2A, 3A, 4A, 5A). The average peak frequencies for each condition are shown in Table 1 and were all slightly different, varying from a low of $\sim 1 \mathrm{~Hz}$ during natural SWS to a high of $2 \mathrm{~Hz}$ during the deactivated state in urethane anesthesia. A one-way ANOVA revealed a significant difference of respiration rate across conditions $\left(F_{(2,21)}=24.52\right.$, $p<0.05)$. Using a Tukey's HSD for post hoc comparisons, these differences were significant for each group compared with each other $(p<0.05)$.

In contrast to the clean and tightbandwidth signals for respiration, LFPs from neocortical and hippocampal sites showed more broadband characteristics (Figs. 2B, 3B, 4B, 5B), with the largest (and typically only) power peak corresponding to the $\mathrm{SO}$, which ranged on average between 1 and $1.5 \mathrm{~Hz}$ across conditions (Table 1). The peak SO signal was highly consistent across all forebrain sites within experiments (especially for cortex and hippocampus), so only one peak frequency was reported across all sites. A one-way ANOVA revealed a significant difference of the frequency of $\mathrm{SO}$ across conditions $\left(F_{(2,21)}=7.82, p<0.05\right)$. A
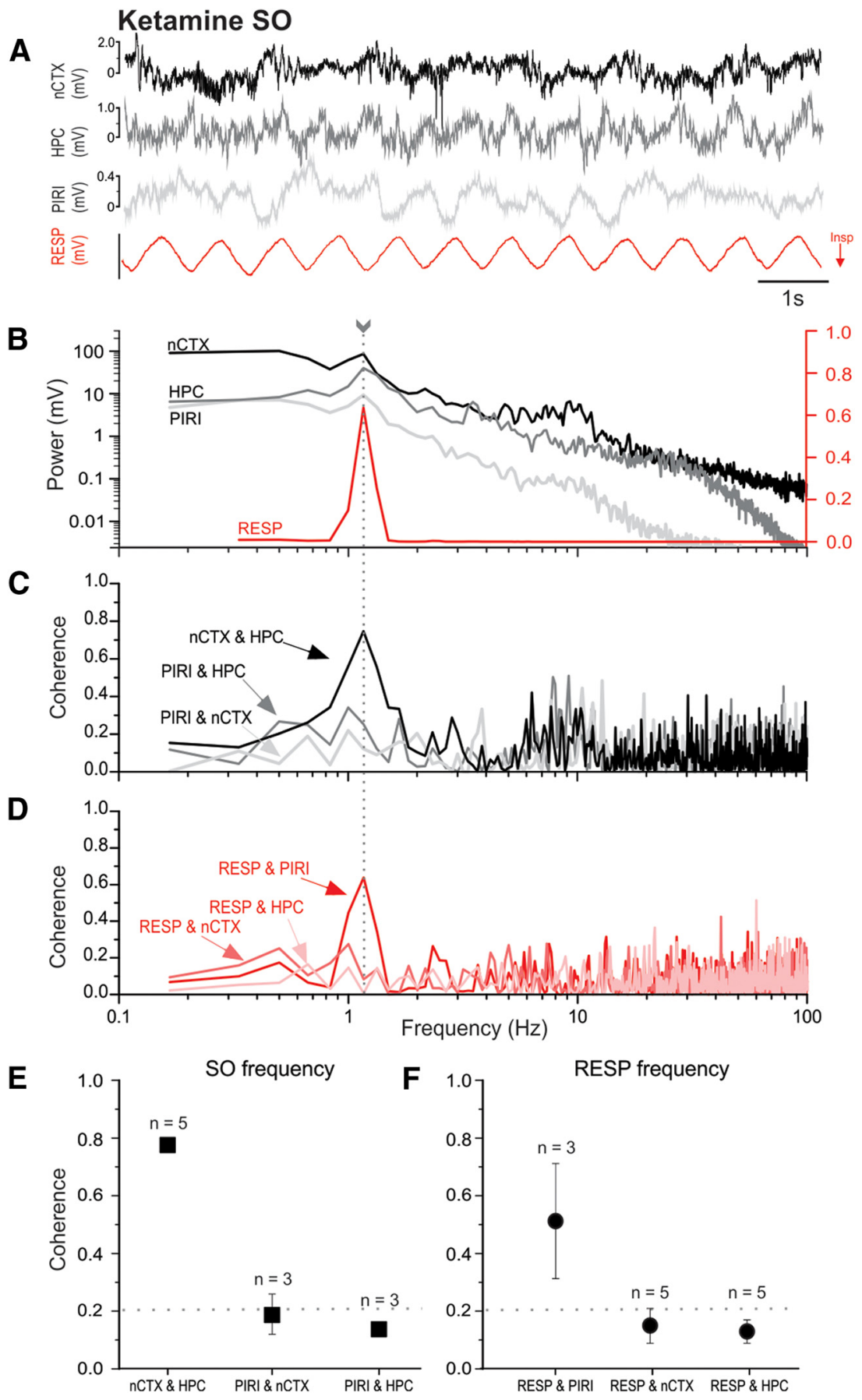

Figure 3. Lack of neocortical and hippocampal coupling to the respiratory cycle under ketamine-xylazine anesthesia at matching fundamental frequencies. $\boldsymbol{A}$, A representative example of simultaneously recorded LFP traces from nCTX, HPC, and PIRI sites, together with thermistor readings from the nostril (RESP, with inspiration shown as downward deflections). $\boldsymbol{B}$, Overlaid power spectral plots from periods (including those shown in $\boldsymbol{A}$ ) demonstrate matching peak frequencies (fundamentals) for $\mathrm{SO}$ and RESP signals. The arrow with the dotted line indicates the matching cortical and respiratory frequency. The logarithmic scale for brain LFP power is shown on the left side and the power of the respiratory signal is displayed on the linear scale to the right. Low-frequency, $<0.33 \mathrm{~Hz}$, near-DC frequencies were truncated from the respiratory plot for ease of visualization. C, Coherence spectra for brain-derived LFP signal pairs demonstrating high coupling across neocortical and hippocampal sites. D. Coherence spectra for the respiratory signal to each brain LFP recording demonstrating low coupling at the respiratory fundamental, while at the same shared frequency, which demonstrates that despite a common fundamental, the respiratory signal was only coherent with piriform activity. All spectra shown $(\boldsymbol{B}-\boldsymbol{D})$ are from the same $1 \mathrm{~min}$ sample that includes the raw sample shown in $\boldsymbol{A} . \boldsymbol{E}, \boldsymbol{F}$, Average coherence $( \pm \mathrm{SEM})$ for all signal pairs for data samples demonstrating matched peak frequency for $\mathrm{SO}$ and RESP signals. The SO, embodied by high nCTX and HPC coherence, emerges as separate process from respiratory activity, indicated by high RESP and PIRI coherence, even when cycling fundamentals are the same. Respiratory (circles) comparisons were separated from LFP (square) signal pairings for ease of visualization. The numbers of samples for averages are displayed above the symbols. The averages resulted from samples collected from a subset of five experiments incidentally all under ketamine-xylazine anesthesia. The horizontal dotted line indicates the threshold for significant coherence, $>0.21$. 
A

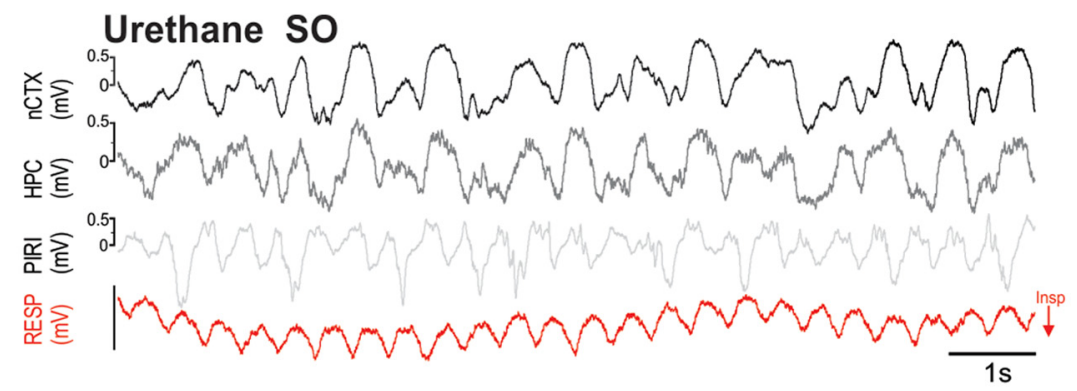

B

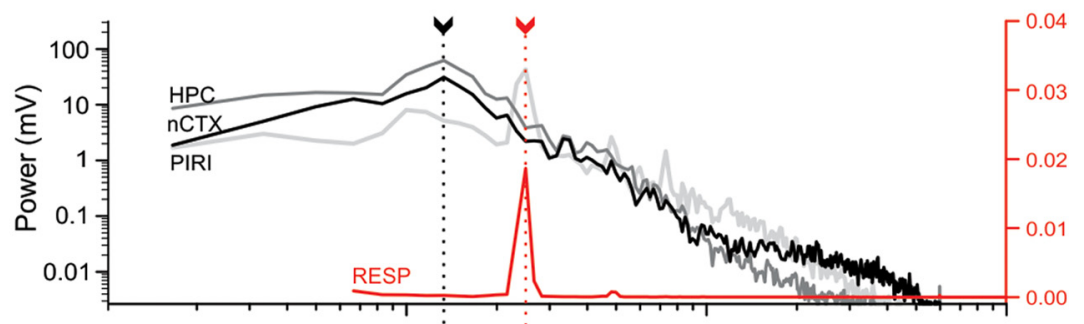

C

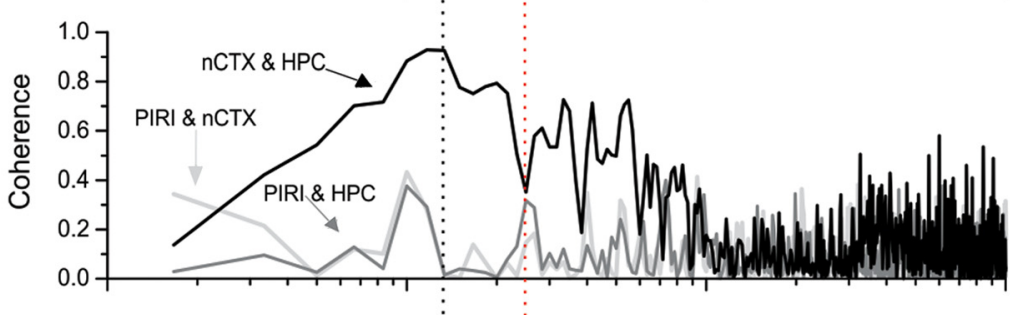

D

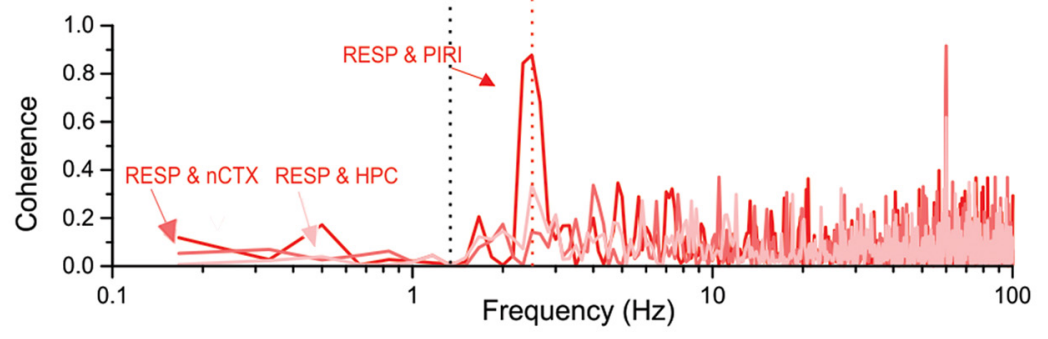

E
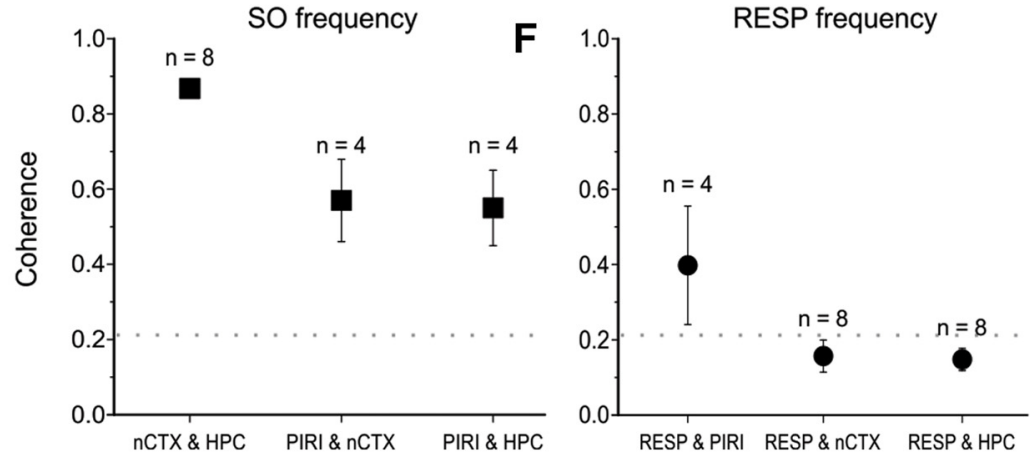

Figure 4. Lack of frequency matching of neocortical/hippocampal and respiratory signals and lack of neocortical/hippocampal coupling to the respiratory cycle under urethane anesthesia. $A$, Representative example of simultaneously recorded LFP traces from nCTX, HPC, and PIRI sites, together with thermistor readings from the nostril (RESP, with inspiration shown as downward deflections). B, Overlaid power spectral plots (from periods including those shown in $A$ ) demonstrate different peak frequencies (fundamentals) when comparing brain-derived LFPs and the respiratory signal. The only LFP signal with a strong peak at the respiratory frequency was that from the piriform cortex. The piriform also demonstrates a separate lower frequency fundamental frequency that overlaps with the neocortical/hippocampal SO peak. Arrows with dotted lines indicate peak frequencies corresponding to cortical SO (black) and respiration (red) frequencies. The logarithmic scales for brain LFP power are shown on the left and the power of the respiratory signal is displayed on the linear scale to the right. Low-frequency, $<0.67 \mathrm{~Hz}$, near-DC-related activity was truncated from the respiratory plot for ease of visualization. C, Coherence spectra for brain-derived LFP signal pairs illustrating high coupling of all LFP pairs around the SO peak, especially across neocortical and hippocampal sites. $\boldsymbol{D}$, Coherence spectra for the respiratory signal to each brain site demonstrating low coupling at the RESP fundamental except at the piriform site. All spectra shown $(\boldsymbol{B}-\boldsymbol{D})$ are from the same $1 \mathrm{~min}$ sample that includes the raw sample shown in $\boldsymbol{A}$. $\boldsymbol{E}$, Average coherence ( \pm SEM) for brain-derived LFP signal pairs as noted across experiments at the SO peak. All sites, especially nCTX to HPC, demonstrate strong pairwise coherence at $S 0$. $F$, Average coherence $( \pm S E M)$ across experiments at the respiration peak frequency for each brain site paired to the respiratory signal. Only the piriform site was significantly coupled to respiration. For both $\boldsymbol{E}$ and $\boldsymbol{F}$, the numbers of subjects used in each average are displayed above the symbols. The horizontal dotted line indicates the threshold for significant coherence, $>0.21$.
Tukey's HSD for post hoc comparisons (at $p<0.05)$ revealed that the only significant difference was between the KET and URE groups, with the SO fastest in KET and slowest in URE. The SO for natural sleep was not significantly different from that of either KET or URE $(p>0.05)$.

More importantly, however, the fundamental frequencies of RESP and the SO were mismatched in all conditions. At the group level, using an independent $t$ test, the average frequencies of RESP and SO were significantly different in both the $\operatorname{URE}\left(t_{(14)}=11.73, p<0.05\right)$ and natural sleep $\left(t_{(6)}=5.27, p<0.05\right)$ conditions. Although group means for RESP and SO frequencies were not significantly different under KET conditions (independent two-sample $t$ test; $\left.t_{(30)}=1.83, p>0.05\right)$, when assessed as a within-subjects design, the absolute differences between the SO and RESP peak frequencies were significantly different from zero for KET, as well as the URE and natural sleep conditions (Table 1). This suggests that on a case-by-case (and also sample-bysample) basis, the respiratory cycle has an entirely different frequency than the SO signal that predominates LFP recordings in the brain.

In contrast to LFP recordings from cortical and hippocampal sites, PIRI LFPs recorded during URE conditions often showed bimodal peaks, one of which matched the respiration cycle frequency and the other overlapping with corticohippocampal SO peaks (Fig. 4B). This suggests that PIRI population activity could be expressed at multiple slow frequencies, including the respiratory cycle, during SWS-like states.

\section{Frequency coupling of respiration and LFP signals}

Despite the nonoverlapping frequencies corresponding to the respiration and cerebral SO signals outside of primary olfactory cortex, we assessed the possibility that respiratory to LFP coupling could still exist at both the respiration and SO peak frequencies, especially given the wider bandwidth of SO LFP signals and the partial overlap of peak frequencies between RESP and the SO. This was done using coherence analysis in each experiment.

Ketamine-xylazine anesthesia

The highest (and the only significant) coupling between the fundamental of the respiration signal $(1.33 \pm 0.11 \mathrm{~Hz}, n=3)$ was to the piriform site with an average coherence of $0.55 \pm 0.15$. Importantly, and as shown in the example in Figure $2 D$ 
(and on average in Fig. $2 F$ ), the respiration signal was not significantly coherent with neocortical LFP sites $(0.15 \pm 0.03)$. The average coherence of respiration to hippocampal LFP just barely crossed the threshold of significance $(0.22 \pm 0.05)$, suggesting at best only very weak coupling. Indeed, across all experiments, there were only 2 cases of 12 that showed a significant value $(>0.2)$ of coherence. However, significant coherent coupling between cortical and hippocampal signals $(0.50 \pm 0.08)$ was observed at the RESP fundamental $(1.41 \pm 0.07 \mathrm{~Hz}, n=12)$ on average. Table 2 shows a breakdown of every pairwise comparison at the peak respiratory frequency.

Conversely, and as we have reported previously (Sharma et al., 2010), the fundamental frequency corresponding to the SO $(1.48 \pm 0.10 \mathrm{~Hz}, n=12)$ was highly coherent across the neocortex and hippocampus $(0.78 \pm 0.05)$. However, and as explicitly shown in Figure $4 C$ (and on average in Fig. $4 E$ ), neither cortical or hippocampal sites showed significant coupling with PIRI at either the RESP or SO peak frequencies. Instead, the PIRI activity was the only brain-derived signal that demonstrated any coupling to respiration at the SO frequency $(0.38 \pm 0.14)$. Table 3 shows a breakdown of every pairwise comparison at the peak SO frequency.

Comparing the coupling profiles across both bandwidths corresponding to the SO and respiration, it is clear that the high coherence of the cortical to hippocampal LFP signals at both respiration and SO peak frequencies is likely due to the large SO bandwidth which overlaps, but is not coherent with, the fundamental frequency of the respiratory signal (Fig. 2B,D). To explore this further, we selected specific cases in which there was perfect overlap of the peak frequencies of SO and respiration-related activities. This occurred in five samples in four subjects (of a total of eight subjects exclusively within the KET condition). Figure 3 shows an example of such overlap. Despite this frequency matching (mean frequency $1.43 \pm 0.08 \mathrm{~Hz}, n=5$ ), the coherence of the respiratory signal to both cortical and hippocampal signals was nevertheless low and nonsignificant $(0.15 \pm 0.06$ and $0.13 \pm$ 0.04 , respectively), whereas the coupling of respiration to piriform recordings was still significant $(0.51 \pm 0.20)$ and similar to that reported for the overall pooled value, as reported in Table 1. As with unmatched peak frequencies, the coupling of hippocampal and cortical signals at this same frequency remained high and significant $(0.78 \pm 0.01)$, whereas piriform coupling to either hippocampal or neocortical sites was low and nonsignificant.
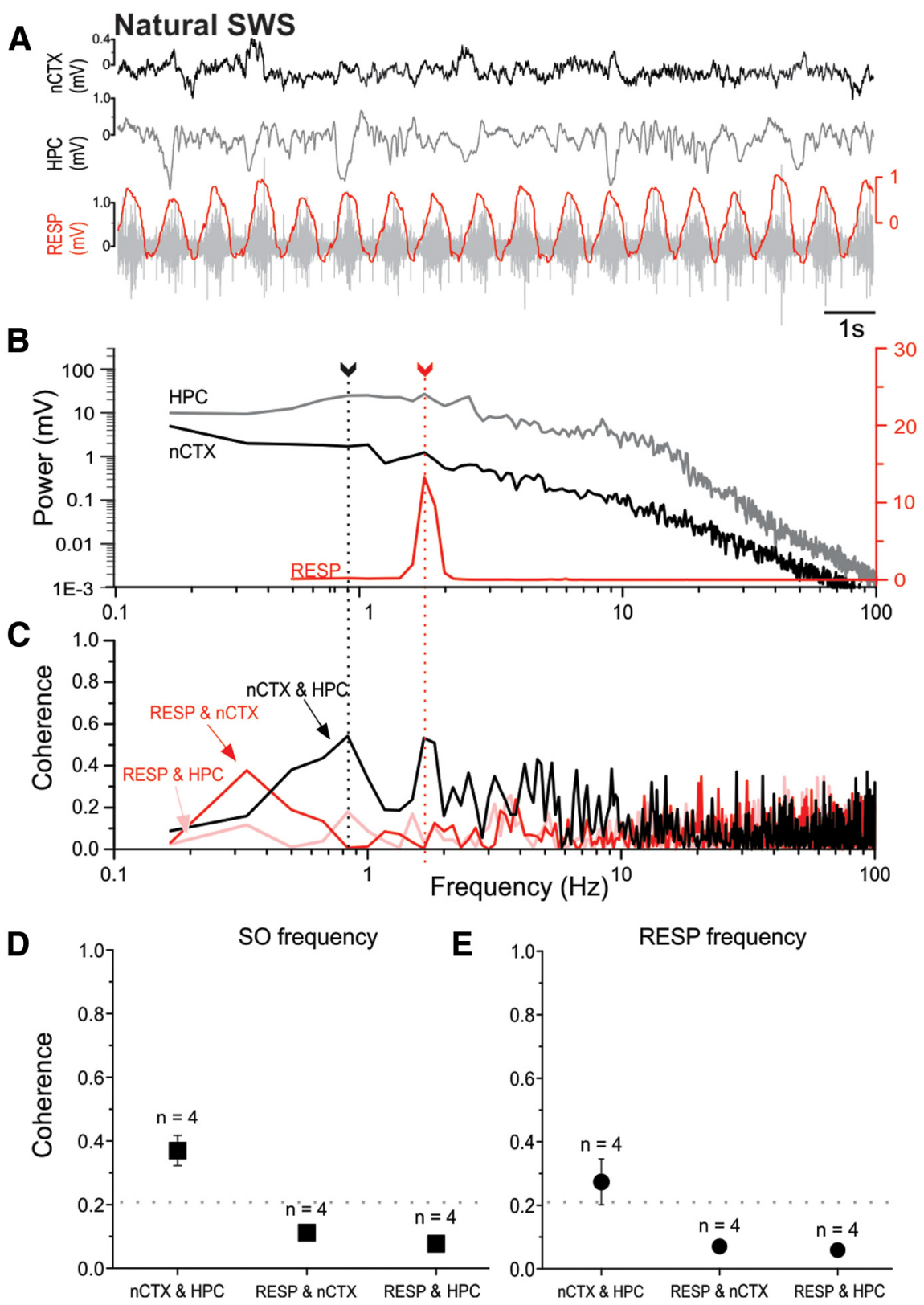

Figure 5. Lack of frequency matching of neocortical/hippocampal and respiratory signals and lack of neocortical/hippocampal coupling to the respiratory cycle during natural sleep. $\boldsymbol{A}$, Representative example of simultaneously recorded raw LFP traces from $\mathrm{nCTX}$ and HPC sites, together with EMG readings from intercostal implantations (RESP; gray). Overlaying the raw EMG is its RMS envelope (red). $\boldsymbol{B}$, Overlaid power spectral plots from periods including those shown in $\boldsymbol{A}$ demonstrate different peak frequencies (fundamentals) when comparing brain-derived LFPs and the respiratory signal. Arrows with dotted lines indicate peak frequencies corresponding to cortical SO (black) and respiration (red) frequencies. The logarithmic scales for brain LFP power are shown on the left and the power of the respiratory signal is displayed on the linear scale to the right. Low-frequency, $<0.5 \mathrm{~Hz}$, near-DC-related activity was truncated from the respiratory plot for ease of visualization. C, Coherence spectra for all signal pairs illustrating high $\mathrm{nCTX}$ and HPC coupling across the SO bandwidth (and including the respiratory fundamental) but a lack of coupling of RESP with $\mathrm{nCTX}$ and HPC signals. Spectra shown $(\boldsymbol{B}, \boldsymbol{C})$ are from the same 1 min sample that includes the raw sample shown in $\boldsymbol{A}$. $\boldsymbol{D}$, Average coherence ( \pm SEM) for signal pairs at the $S 0$ peak. Only the HPC and nCTX signals are significantly coherent, whereas respiration does not demonstrate coupling with the neocortical-hippocampal SO. $\boldsymbol{E}$, Average coherence ( \pm SEM) across experiments at the respiration peak frequency. As for the SO, only the HPC and nCTX signals were significantly coherent, whereas respiration does not demonstrate coupling with either brain site. For both $\boldsymbol{D}$ and $\boldsymbol{E}$, the numbers of subjects for averages are displayed above the symbols. The horizontal dotted line indicates the threshold for significant coherence, $>0.21$.

\section{Urethane anesthesia}

The respiratory coupling trend under urethane was similar to that reported for KET. The only significant coupling between the respiratory signal and LFP recordings at the fundamental frequency of respiration $(2.10 \pm 0.14 \mathrm{~Hz}, n=4)$ was between the 
Table 1. Across-conditions summary for $\mathrm{SO}$ and respiration peak frequencies

\begin{tabular}{llll}
\hline Condition & SO peak $(\mathrm{Hz})$ & RESP peak $(\mathrm{Hz})$ & SO - RESP within-subjects difference \\
\hline KET & $1.48 \pm 0.10$ & $1.41 \pm 0.07$ & $0.238 \pm 0.056^{* *}$ \\
URE & $1.01 \pm 0.06$ & $2.14 \pm 0.09^{*}$ & $1.132 \pm 0.068^{* *}$ \\
Sleep & $1.23 \pm 0.05$ & $1.76 \pm 0.09^{*}$ & $0.525 \pm 0.082^{* *}$ \\
\hline
\end{tabular}

All values are mean \pm SEM.

${ }^{*} p<0.001$, independent two-sample between-groups $t$ test of $S 0$ and RESP means.

${ }^{* *} p<0.001 ;{ }^{* * *} p<0.05$, one-tailed confidence interval (single-sample $t$ test).

Table 2. Coherence at RESP fundamental frequency during KET

\begin{tabular}{llll}
\hline Site & RESP & nCTX & HPC \\
\hline $\operatorname{RESP}(n=12)$ & N/A & & \\
$\operatorname{nCTX~}(n=12)$ & $0.15 \pm 0.03$ & N/A & \\
HPC $(n=12)$ & $0.22 \pm 0.05^{*}$ & $0.50 \pm 0.07^{*}$ & N/A \\
$\operatorname{PIRI}(n=3)$ & $0.55 \pm 0.15^{*}$ & $0.16 \pm 0.04$ & $0.14 \pm 0.03$ \\
\hline
\end{tabular}

N/A, Not applicable.

${ }^{*}$ Above significance threshold $(>0.21)$.

Table 3. Coherence at SO fundamental frequency during KET

\begin{tabular}{llll}
\hline Site & RESP & nCTX & HPC \\
\hline $\operatorname{RESP}(n=12)$ & N/A & & \\
$\operatorname{nCTX}(n=12)$ & $0.19 \pm 0.05$ & N/A & \\
$\operatorname{HPC}(n=12)$ & $0.18 \pm 0.04$ & $0.78 \pm 0.05^{*}$ & N/A \\
$\operatorname{PIRI}(n=3)$ & $0.38 \pm 0.14^{*}$ & $0.16 \pm 0.08$ & $0.12 \pm 0.06$ \\
\hline
\end{tabular}

N/A, Not applicable.

${ }^{*}$ Above significance threshold $(>0.21)$.

Table 4. Coherence at RESP fundamental frequency during deactivated URE

\begin{tabular}{llll}
\hline Site & RESP & nCTX & HPC \\
\hline $\operatorname{RESP}(n=8)$ & N/A & & \\
$\operatorname{nCTX~}(n=8)$ & $0.16 \pm 0.04$ & N/A & \\
HPC $(n=8)$ & $0.15 \pm 0.03$ & $0.53 \pm 0.05^{*}$ & N/A \\
$\operatorname{PIRI}(n=4)$ & $0.40 \pm 0.16^{*}$ & $0.12 \pm 0.03$ & $0.21 \pm 0.01$ \\
\hline
\end{tabular}

N/A, Not applicable.

*Above significance threshold $(>0.21)$.

respiratory signal and the piriform cortex $(0.40 \pm 0.16)$. Again, although the respiratory cycle was not significantly coherent with either cortical or hippocampal signals (as shown in in the example in Fig. $4 D$ and on average in Fig. $4 F$ ), significant coherent coupling $(0.53 \pm 0.05)$ was observed between cortical and hippocampal signals at the breathing fundamental $(2.14 \pm 0.09 \mathrm{~Hz}$, $n=8)$. Table 4 shows a breakdown of all average pairwise coherence values at the respiratory fundamental across signals. Independent two-sample $t$ testing of mono versus bipolar hippocampal-neocortical coherence demonstrated that electrode configurations were not significantly different during the nREMlike state (nREM-like: $t_{(6)}=-1.00, p>0.10$ ). This was also the case for hippocampal-respiratory coupling during nREM-like states (nREM-like: $t_{(6)}=-1.43, p>0.10$ ).

Again similarly to KET, the pattern of LFP coherence at the SO peak frequency was similar across signals in the URE condition. As shown in the example in Figure $4 C$ (and on average in Figure $4 E$ ), in addition to the previously reported (Wolansky et al., 2006; Sharma et al., 2010) high and significant coherence $(0.87 \pm 0.02)$ between cortical and hippocampal signals at the SO frequency $(1.01 \pm 0.06, n=8)$, the piriform signal was also significantly coherent with both the neocortical $(0.57 \pm 0.11)$ and hippocampal signals $(0.55 \pm 0.10)$ at the SO peak $(0.93 \pm 0.07 \mathrm{~Hz}, n=4)$. Table 5 shows all pairwise signal comparisons at the SO peak frequency. Again importantly for our present findings, our data
Table 5. Coherence at SO fundamental frequency during deactivated URE

\begin{tabular}{llll}
\hline Site & RESP & nCTX & HPC \\
\hline $\operatorname{RESP}(n=8)$ & $\mathrm{N} / \mathrm{A}$ & & \\
$\mathrm{nCTX}(n=8)$ & $0.12 \pm 0.03$ & $\mathrm{~N} / \mathrm{A}$ & \\
$\mathrm{HPC}(n=8)$ & $0.12 \pm 0.03$ & $0.87 \pm 0.02^{*}$ & $\mathrm{~N} / \mathrm{A}$ \\
$\operatorname{PIRI}(n=4)$ & $0.06 \pm 0.03$ & $0.57 \pm 0.11^{*}$ & $0.55 \pm 0.10^{*}$ \\
\hline
\end{tabular}

N/A, Not applicable.

${ }^{*}$ Above significance threshold $(>0.21)$.

Table 6. Coherence at RESP fundamental frequency during SWS

\begin{tabular}{lll}
\hline Site & RESP & nCTX \\
\hline $\operatorname{RESP}(n=4)$ & N/A & \\
$\operatorname{nCTX}(n=4)$ & $0.07 \pm 0.01$ & N/A \\
$\operatorname{HPC}(n=4)$ & $0.06 \pm 0.01$ & $0.27 \pm 0.07^{*}$ \\
\hline
\end{tabular}

N/A, Not applicable.

${ }^{*}$ Above significance threshold $(>0.21)$.

Table 7. Coherence at SO fundamental frequency during SWS

\begin{tabular}{lll}
\hline Site & RESP & nCTX \\
\hline $\operatorname{RESP}(n=4)$ & N/A & \\
$\operatorname{nCTX}(n=4)$ & $0.11 \pm 0.01$ & N/A \\
$\operatorname{HPC}(n=4)$ & $0.08 \pm 0.01$ & $0.37 \pm 0.05^{*}$ \\
\hline
\end{tabular}

$\mathrm{N} / \mathrm{A}$, Not applicable.

${ }^{*}$ Above significance threshold $(>0.21)$.

show a fundamental lack of coupling of the respiratory signal to forebrain LFP other than to the piriform site.

Natural sleep

In natural sleeping conditions, the only pair of significantly coherent signals were those at the cortical and hippocampal sites at the SO fundamental (Fig. 5C-F). At the peak frequency of the SO $(1.23 \pm 0.05, n=4)$, the average corticohippocampal coherence was $0.37 \pm 0.05$. Similarly, at the respiratory peak frequency $(1.76 \pm 0.09, n=4)$, the average corticohippocampal coherence was also significant at $0.27 \pm 0.07$. However, and as shown in Tables 6 and 7, the respiratory signal was not significantly coupled to either the cortical or hippocampal sites at either the respiration or SO peak frequencies. Although we did not have piriform recordings during natural sleep, our results concerning the lack of respiratory-LFP coupling at neocortical and hippocampal sites were entirely consistent with our reported data for both ketamine and urethane anesthesia (cf. Figs. $2 F, 3 E, 4 F, 5 E$ ).

\section{Frequency coupling of respiration across state changes}

Although our main purpose was to evaluate the degree of correspondence of the respiratory cycle to the cortical and hippocampal SO, we also had the opportunity to compare respiratory coupling between these signals during and across the occurrence of spontaneous brain state changes from slow-wave to activated (REM and REM-like) patterns during both urethane anesthesia and natural sleep. During REM-like states under urethane anesthesia, the coupling of the respiratory signal at its fundamental $(2.26 \pm 0.12 \mathrm{~Hz}: n=8)$ to PIRI, nCTX, and HPC signals increased by $151 \pm 29 \%$ on average (Fig. $6 A$ ). Table 8 illustrates the site pair coherence values. All pairwise increases of respiratory frequency coherence were significant (1-tailed $t$ tests RESP to: PIRI, $t_{(3)}=2.55, p<0.05 ; \operatorname{nCTX}_{(7)}=4.78, p<0.01 ; \mathrm{HPC}, t_{(7)}$ $=3.00, p<0.01)$. During natural sleep, there was a similar increase in coupling of the respiratory signal at its fundamental $(1.75 \pm 0.03 \mathrm{~Hz})$ to brain LFPs during REM. However, this increase (averaging $158 \pm 72 \%$ across sites) was not significant 
(1-tailed paired $t$ tests RESP: nCTX, $t_{(3)}=$ $2.08, p>0.05$; HPC, $\left.t_{(3)}=1.41, p>0.05\right)$. Table 9 contains the site pair coherence values and Figure $6 B$ illustrates respiratory coherence during the REM state.

\section{Discussion}

Our results indicate that the fundamental frequencies corresponding to respiration and the cerebral SO during deactivated (nREM and nREM-like) states are neither identical nor coupled. This finding applies to ketamine-xylazine and urethane anesthesia in addition to natural sleep. Importantly, we found no evidence that, as proposed previously (Fontanini et al., 2003; Fontanini and Bower, 2006), there is any coupling or entrainment of respiratory-related activity broadly throughout the forebrain during SO states except for the primary olfactory (piriform) cortex. In contrast, during activated (REM and REM-like) states, the coupling of forebrain signals to respiratoryrelated activity may be enhanced across most forebrain regions.

\section{Cortical slow-waves and olfactory rhythms}

The suggested coupling of slow-wave brain activity to resting nasal respiration stems from the initial finding of nonodorant respiratoryrelated rhythmic coupling of olfactory bulb and piriform cortex activity (Fontanini et al., 2003). This work suggested that olfactory receptors in the nasal mucosum, at least during ketamine anesthesia, were sensitive to the rhythmic passage of inspired air and that this phasic respiratory excitation subsequently entrained activity in the olfactory bulb and then piriform cortex. Although slow activity in piriform cortex could be independently decoupled from respiration by tracheotomy, entrainment was reinstated by blowing air through the nasal passage. Indeed, respiratory entrainment was also demonstrated to be more pronounced during deep (as opposed to light) anesthesia, when SO activity tends to be more prominent throughout the cerebrum (Fontanini and Bower, 2005). This suggested that, in the olfactory cortex, independently generated slow oscillatory activity was subject to sensory entrainment, especially during slowwave states. The investigators proposed that this entrainment was also likely to spread more broadly throughout the cerebrum due to the prominent connections of piriform areas to both frontal and parahippocampal/hippocampal cortices (Fontanini et al., 2003; Fontanini and Bower, 2006). By measuring LFP activity directly together with the ongoing respiratory cycle and using high-resolution frequency-specific coupling measures across signals in the present study, we were able to assess this proposition directly. We have shown conclusively, across two different anesthetic agents and during natural sleep, that such respiratory coupling does not occur in either frontal or hippocampal cortices during slow-wave states. However, we did confirm that piriform activity under anesthesia does couple to respiration, as was observed previously (Fontanini et al., 2003; Fontanini and Bower, 2005), but also that piriform activity may independently couple with cerebral SO.

Our conclusive findings were related to both low coupling measures and, perhaps more importantly, the finding that power spectral fundamentals corresponding to the respiratory cycle and the cerebral SO were entirely different. Even in the few cases (all limited to the KET condition) where RESP and SO fundamentals happened to
Table 8. Coherence at RESP fundamental frequency during activated URE

\begin{tabular}{llll}
\hline Site & RESP & nCTX & HPC \\
\hline $\operatorname{RESP}(n=8)$ & N/A & & \\
$\operatorname{nCTX}(n=8)$ & $0.49 \pm 0.06^{*}$ & N/A & N/A \\
HPC $(n=8)$ & $0.36 \pm 0.08^{*}$ & $0.30 \pm 0.08^{*}$ & $0.38 \pm 0.11^{*}$ \\
$\operatorname{PIRI}(n=4)$ & $0.83 \pm 0.06^{*}$ & $0.47 \pm 0.04^{*}$ & \\
\hline
\end{tabular}

$\mathrm{N} / \mathrm{A}$, Not applicable.

${ }^{*}$ Above significance threshold $(>0.21)$.

Table 9. Coherence at RESP fundamental frequency during REM sleep

\begin{tabular}{lll}
\hline Site & RESP & nCTX \\
\hline $\operatorname{RESP}(n=4)$ & N/A & \\
$\operatorname{nCTX~}(n=4)$ & $0.16 \pm 0.05$ & N/A \\
HPC $(n=4)$ & $0.14 \pm 0.05$ & $0.17 \pm 0.03$
\end{tabular}

$\mathrm{N} / \mathrm{A}$, Not applicable.

${ }^{*}$ Above significance threshold $(>0.21)$.

be exactly matched, low coherence was consistently found between the respiratory signal and cerebral LFPs located outside of piriform cortex. These results suggest that the hippocampal and neocortical $\mathrm{SO}$ are entirely separate and independent processes from respiratory activity during slow-wave states. Interestingly, and indicative of the broad bandwidth of SO activity (even during more stationary conditions such as anesthesia), neocortical-hippocampal coupling extended to include the peak fundamental of the respiratory signal, even though the respiratory signal itself was not coherent with signals from either of these sites.

\section{Active versus resting olfactory-related oscillatory synchronization}

The synchronization of oscillatory rhythms in olfactory systems has long been thought to be an active computational process device for odor perception and recognition (Freeman, 1975; Kay et al., 2009). It is thus perhaps not surprising, especially based upon anatomical connectivity, that fast ( $\beta$ /gamma) odor-related synchrony and oscillatory coupling can also exist among the piriform, prefrontal, and hippocampal cortices during direct olfactory stimulation, as well as during learning-related odor sampling 
(Price, 1985; Cinelli et al., 1987; Clugnet and Price, 1987; Price et al., 1991; Vanderwolf, 1992; Heale et al., 1994; Martin et al., 2006; Kay et al., 2009). Although olfactory structures (presumably including olfactory receptors themselves) are excited in phase with inspiration during unconsciousness when breathing plain air (Wilson, 1998; Fontanini et al., 2003; Fontanini and Bower, 2005), the present study clearly shows that the slow, and likely also the fast, respiratory-related component does not appear to be further transmitted downstream during slow-wave states.

Given that brain state is well known to modulate frequencydependent neural coupling across forebrain regions, a remaining question concerns how respiration and the SO might be independently expressed and coupled in these regions across states. Interestingly, a recent study using urethane-anesthetized rats suggested that slow-frequency $(<5 \mathrm{~Hz})$ coupling of piriform to olfactory bulb activity during nREM-like states was lower than during activated (REM-like) states, whereas piriform to hippocampal coupling increased (Wilson and Yan, 2010). This is of significance because the expression and coupling of olfactory bulb activity to the respiratory cycle was consistently high across state changes, suggesting that, in contrast to Fontanini and Bower (2005), piriform activity couples less to respiration and more to forebrain SO during slow-wave states. Unlike the present study, however, the frequency resolution $(4.8 \mathrm{~Hz})$ of the Wilson and Yan (2010) work was too broad to permit a separation of the influence of the SO versus respiration fundamentals per se. However, in parallel to suggestions made by Wilson and Yan (2010) and perhaps also earlier work by Freeman (1959), we also observed enhancements of respiratory coupling to activity piriform cortex (and beyond) across deactivated to activated brain states. Although our results appear to be in agreement with the idea that effective corticocortical connectivity is higher during activated, as opposed to during deactivated (nREM), states (Massimini et al., 2005), they contrast directly those of Fontanini and Bower (2005), who suggested that respiratory to piriform coupling was stronger (at least during KET anesthesia) during deeper anesthetic slow-wave states.

State dependencies aside, a potential source of coupling error that we occasionally encountered was due to signal referencing problems. Monopolar brain LFP signals can show prominent respiration-induced artifacts due to inappropriate or improperly grounded reference electrode locations that are positioned to pick up respiratory signals themselves (e.g., due to respiratory movements). In our own experiments, we determined that any continuous electrical contact with the nasally placed thermistor was reflected in LFP recordings as a strong respiratory rhythm in our monopolar LFP recordings that were referenced to either the animal or the stereotaxic apparatus. These problems were eliminated by staggered depth bipolar recording montages for LFP or by insulating the thermistor from any electrical contact with the animal. Although we cannot definitively exclude these grounding problems in the two ketamine experiments in which we did see significant (i.e., $>0.21$ ) respiratory to forebrain coherence, based on the consistency of the bulk of our findings (and especially across different conditions), we feel that this may likely be the case.

Although we evaluated data from a broad array of conditions and central cerebral sites, we did not include isoflurane anesthesia nor did we obtain piriform recordings in natural sleep. It is our experience (Dickson et al., 2007), that cerebral activity at adequate anesthetic levels of isoflurane is better described by patterns of burst suppression as opposed to sleep-like slow-wave activity. Furthermore, isoflurane also does not allow spontaneous alternations of state that we readily observe with adequate urethane anesthesia (Clement et al., 2008). Regarding piriform recordings during natural sleep, it was our major intent to simply evaluate the potential for respiratory coupling to cerebral sites outside of the piriform area, as proposed by Fontanini and Bower (2006). As specified, data from these animals, used in a prior study to evaluate respiratory modulation across sleep states $(\mathrm{Pa}-$ gliardini et al., 2012), did not include a piriform recording site. However, they did include both neocortical and hippocampal placements that allowed us to analyze coupling of respiratory measures with those sites during natural slow-wave and REM states. Although the coupling of piriform activity across naturally sleeping brain states is an important question, it was not the primary concern of the present study.

\section{Conclusion}

Here, we have evaluated directly the validity of respiratoryrelated activity as an offline cerebral SO-entraining mechanism. Given that excitatory influences can induce entrainment of the SO (Amzica and Steriade, 1995; Petersen et al., 2003; MacLean et al., 2005; Massimini et al., 2007; Gao et al., 2009; Vyazovskiy et al., 2009; Ozen et al., 2010; Zhang et al., 2011; Greenberg and Dickson, 2013), we were interested in validating the proposal of olfactory entrainment of SO. We have shown conclusively, across the ketamine-xylazine, urethane, and natural sleep conditions, that the $\mathrm{SO}$ repeatedly emerges as a distinct and separate process from respiration and that this separation was robust even when both overlapped in frequency. In contrast, and as shown previously, piriform cortex activity was reliably coupled to respiratory inspiration, although a component of its activity could overlap and couple with the cerebral SO. These results fit well with other work suggesting that the origin and generation of ongoing offline cerebral SO is not a product of (nor is it influenced by) inspiratory respiratory activity (Freeman, 1959; Heale et al., 1994). Instead, they fit with an SO mechanism as an intrinsic emergent property of a deafferented neural network (Timofeev and Steriade, 1996; Sanchez-Vives and McCormick, 2000; Timofeev et al., 2000; Dickson et al., 2003).

\section{References}

Amzica F, Steriade M (1995) Disconnection of intracortical synaptic linkages disrupts synchronization of a slow oscillation. J Neurosci 15:46584677. Medline

Born J, Rasch B, Gais S (2006) Sleep to remember. Neuroscientist 12:410 424. CrossRef Medline

Buzsáki G (1989) Two-stage model of memory trace formation: A role for "noisy" brain states. Neurosci 31:551-570. CrossRef Medline

Buzsáki G (1998) Memory consolidation during sleep: a neurophysiological perspective. J Sleep Res 7:17-23. CrossRef Medline

Buzsáki G (2006) Rhythms of the brain. Oxford: OUP.

Buzsáki G, Draguhn A (2004) Neuronal oscillations in cortical networks. Science 304:1926-1929. CrossRef Medline

Chaput M, Holley A (1980) Single unit responses of olfactory bulb neurones to odour presentation in awake rabbits. J Physiol (Paris) 76:551-558. Medline

Cinelli AR, Ferreyra-Moyano H, Barragan E (1987) Reciprocal functional connections of the olfactory bulbs and other olfactory related areas with the prefrontal cortex. Brain Res Bull 19:651-661. CrossRef Medline

Clement EA, Richard A, Thwaites M, Ailon J, Peters S, Dickson CT (2008) Cyclic and sleep-like spontaneous alternations of brain state under urethane anaesthesia. PLoS One 3:e2004. CrossRef Medline

Clugnet MC, Price JL (1987) Olfactory input to the prefrontal cortex in the rat. Ann N Y Acad Sci 510:231-235. CrossRef

Dickson CT (2010) Ups and downs in the hippocampus: the influence of oscillatory sleep states on "neuroplasticity" at different time scales. Behav Brain Res 214:35-41. CrossRef Medline

Dickson CT, Biella G, de Curtis M (2003) Slow periodic events and their 
transition to gamma oscillations in the entorhinal cortex of the isolated Guinea pig brain. J Neurophysiol 90:39-46. CrossRef Medline

Dickson C, Lo A, Clement E, Mah E, Richard A (2007) Program No. 791.793. Cyclical and sleep-like alternations of brain state are specific to urethane anaesthesia. 2007 Neuroscience Meeting Planner, San Diego, CA: Society for Neuroscience. Online.

Fontanini A, Bower JM (2005) Variable coupling between olfactory system activity and respiration in ketamine/xylazine anesthetized rats. J Neurophysiol 93:3573-3581. CrossRef Medline

Fontanini A, Bower JM (2006) Slow-waves in the olfactory system: an olfactory perspective on cortical rhythms. Trends Neurosci 29:429-437. CrossRef Medline

Fontanini A, Spano P, Bower JM (2003) Ketamine-xylazine-induced slow $(<1.5 \mathrm{~Hz})$ oscillations in the rat piriform (olfactory) cortex are functionally correlated with respiration. J Neurosci 23:7993-8001. Medline

Freeman WJ (1959) Distribution in time and space of prepyriform electrical activity. J Neurophysiol 22:644-665. Medline

Freeman WJ (1975) Mass action in the nervous system. New York: Academic.

Gao L, Meng X, Ye C, Zhang H, Liu C, Dan Y, Poo MM, He J, Zhang X (2009) Entrainment of slow oscillations of auditory thalamic neurons by repetitive sound stimuli. J Neurosci 29:6013-6021. CrossRef Medline

Girardeau G, Zugaro M (2011) Hippocampal ripples and memory consolidation. Curr Opin Neurobiol 21:452-459. CrossRef Medline

Greenberg A, Dickson CT (2013) Spontaneous and electrically modulated spatiotemporal dynamics of the neocortical slow oscillation and associated local fast activity. Neuroimage 83:782-794. CrossRef Medline

Heale VR, Vanderwolf CH, Kavaliers M (1994) Components of weasel and fox odors elicit fast wave bursts in the dentate gyrus of rats. Behav Brain Res 63:159-165. CrossRef Medline

Kay LM, Beshel J, Brea J, Martin C, Rojas-Líbano D, Kopell N (2009) Olfactory oscillations: the what, how and what for. Trends Neurosci 32:207214. CrossRef Medline

MacLean JN, Watson BO, Aaron GB, Yuste R (2005) Internal dynamics determine the cortical response to thalamic stimulation. Neuron 48:811823. CrossRef Medline

Marshall L, Born J (2007) The contribution of sleep to hippocampusdependent memory consolidation. Trends Cogn Sci 11:442-450. CrossRef Medline

Martin C, Gervais R, Messaoudi B, Ravel N (2006) Learning-induced oscillatory activities correlated to odour recognition: a network activity. Eur J Neurosci 23:1801-1810. CrossRef Medline

Massimini M, Ferrarelli F, Huber R, Esser SK, Singh H, Tononi G (2005) Breakdown of cortical effective connectivity during sleep. Science 309: 2228-2232. CrossRef Medline

Massimini M, Ferrarelli F, Esser SK, Riedner BA, Huber R, Murphy M, Peterson MJ, Tononi G (2007) Triggering sleep slow waves by transcranial magnetic stimulation. Proc Natl Acad Sci US A 104:8496-8501. CrossRef Medline

Mölle M, Born J (2011) Slow oscillations orchestrating fast oscillations and memory consolidation. Prog Brain Res 193:93-110. CrossRef Medline

Ozen S, Sirota A, Belluscio MA, Anastassiou CA, Stark E, Koch C, Buzsáki G (2010) Transcranial electric stimulation entrains cortical neuronal populations in rats. J Neurosci 30:11476-11485. CrossRef Medline

Pagliardini S, Greer JJ, Funk GD, Dickson CT (2012) State-dependent mod- ulation of breathing in urethane-anesthetized rats. J Neurosci 32:1125911270. CrossRef Medline

Paxinos G, Watson C (1998) The rat brain in stereotaxic coordinates, Ed 4. San Diego: Academic.

Petersen CC, Hahn TT, Mehta M, Grinvald A, Sakmann B (2003) Interaction of sensory responses with spontaneous depolarization in layer $2 / 3$ barrel cortex. Proc Natl Acad Sci U S A 100:13638-13643. CrossRef Medline

Price JL (1985) Beyond the primary olfactory cortex: olfactory related areas in the neocortex, thalamus and hypothalamus. Chem Senses 10:239-258. CrossRef

Price JL, Carmichael ST, Carnes KM, Clugnut MC, Kuroda M, Ray JP (1991) Olfactory input to the prefrontal cortex. In: Olfaction: a model system for computational neuroscience (Davis JL, Eichenbaum H, eds), pp 101-120. Boston: MIT.

Sanchez-Vives MV, McCormick DA (2000) Cellular and network mechanisms of rhythmic recurrent activity in neocortex. Nat Neurosci 10:1027-1034.

Sharma AV, Wolansky T, Dickson CT (2010) A comparison of sleeplike slow oscillations in the hippocampus under ketamine and urethane anesthesia. J Neurophysiol 104:932-939. CrossRef Medline

Steriade M, Nuñez A, Amzica F (1993a) Intracellular analysis of relations between the slow $(<1 \mathrm{~Hz})$ neocortical oscillation and other sleep rhythms of the electroencephalogram. J Neurosci 13:3266-3283. Medline

Steriade M, Nuñez A, Amzica F (1993b) A novel slow ( $<1 \mathrm{~Hz}$ ) oscillation of neocortical neurons in vivo: depolarizing and hyperpolarizing components. J Neurosci 13:3252-3265. Medline

Timofeev I, Steriade M (1996) Low-frequency rhythms in the thalamus of intact-cortex and decorticated cats. J Neurophysiol 76:4152-4168. Medline

Timofeev I, Grenier F, Bazhenov M, Sejnowski TJ, Steriade M (2000) Origin of slow cortical oscillations in deafferented cortical slabs. Cereb Cortex 10:1185-1199. CrossRef Medline

Tononi G, Cirelli C (2006) Sleep function and synaptic homeostasis. Sleep Med Rev 10:49-62. CrossRef Medline

Vanderwolf CH (1992) Hippocampal activity, olfaction, and sniffing: an olfactory input to the dentate gyrus. Brain Res 593:197-208. CrossRef Medline

Vyazovskiy VV, Faraguna U, Cirelli C, Tononi G (2009) Triggering slow waves during NREM sleep in the rat by intracortical electrical stimulation: effects of sleep/wake history and background activity. J Neurophysiol 101:1921-1931. CrossRef Medline

Wilson DA (1998) Habituation of odor responses in the rat anterior piriform cortex. J Neurophysiol 79:1425-1440. Medline

Wilson DA, Yan X (2010) Sleep-like states modulate functional connectivity in the rat olfactory system. J Neurophysiol 104:3231-3239. CrossRef Medline

Wolansky T, Clement EA, Peters SR, Palczak MA, Dickson CT (2006) The hippocampal slow oscillation: A novel EEG state and its coordination with ongoing neocortical activity. J Neurosci 26:6213-6229. CrossRef Medline

Zhang Y, Han L, Xiao X, Hu B, Ruan H, Xiong Y (2011) Effects of acoustic stimuli on neuronal activity in the auditory cortex of the rat. Physiol Res 60:687-693. Medline 